\title{
Chronic Pain and Quality of Life in Maintenance Hemodialysis Patients in China: A Multicenter, Cross-Sectional Study
}

\author{
Qian Rao (D) \\ Jing Zeng' \\ Shaoqing Wang ${ }^{2}$ \\ Jue Hao' \\ Menglin Jiang' \\ 'School of Nursing, Chengdu Medical \\ College, Chengdu, People's Republic of \\ China; ${ }^{2}$ Department of Nephrology, The \\ Second Affiliated Hospital of Chengdu \\ Medical College, Chengdu, People's \\ Republic of China
}

Correspondence: Jing Zeng School of Nursing, Chengdu Medical College, No. 60I Tianhui Road, Rongdu Avenue, Chengdu, 610083, People's Republic of China

Tel +0086-28-62308666

Email zengjinger@cmc.edu.cn
Purpose: Patients undergoing maintenance hemodialysis (MHD) frequently experience chronic pain, which can severely affect their quality of life (QOL). The objective of this study was to evaluate the prevalence of chronic pain in MHD patients and examine the factors associated with QOL.

Patients and Methods: A cross-sectional questionnaire-based survey was conducted between October 2020 and April 2021, 1204 MHD patients from nine hemodialysis units were screened for chronic pain in Chengdu, China, and 296 MHD patients with chronic pain were enrolled in this study. We analyzed data on clinicodemographic characteristics, pain interference and severity (Brief Pain Inventory), QOL (Medical Outcomes Study 36-item Short Form Health Survey - mental component summary [MCS] and physical component summary [PCS]), pain self-efficacy (Pain Self-Efficacy Questionnaire), and social support (Social Support Rating Scale).

Results: The prevalence of chronic pain in MHD patients was $26.74 \%$ in this study. The most common areas of pain were lower back (63.5\%), lower limbs $(55.0 \%)$, and head (33.5\%), 36.5\% did not implement any measures to relieve it. Of the patients who did receive pain treatment or medication, $56.9 \%$ reported that the measures they took had less than half of the pain relief. MHD patients with chronic pain had poor QOL based on scores on the MCS $(53 \pm 16.76)$ and PCS $(40.56 \pm 13.81)$. Stepwise multiple regression identified age, financial strain, pain interference, social support, and pain self-efficacy as independent predictors of QOL. Pain self-efficacy was significantly associated with social support ( $r=$ $0.5, \mathrm{p}<0.01)$, MCS $(r=0.69, \mathrm{p}<0.01)$, and PCS $(r=0.8, \mathrm{p}<0.01)$. The mediating effects of pain self-efficacy were $70.31 \%$ on the relationship between social support and MCS, and $75.62 \%$ on the relationship between social support and PCS.

Conclusion: Chronic pain is prevalent and undermanaged in Chinese MHD patients, resulting in worse QOL. Healthcare providers should focus on pain management and the impact of psychosocial factors on patient QOL. Further research should deepen our understanding of how pain self-efficacy mediates the relationship between social support and QOL.

Keywords: chronic pain, maintenance hemodialysis, pain management, quality of life, pain self-efficacy, social support

\section{Plain Language Summary}

In this multicenter cross-sectional study, we evaluated the levels of chronic pain experienced by Chinese patients on maintenance hemodialysis and identified factors associated with their quality of life. Our results indicate that chronic pain is prevalent and undermanaged in these 
patients, and their quality of life is significantly impacted by clinicodemographic characteristics such as age, financial strain and pain interference, as well as psychosocial factors such as social support and pain self-efficacy.

\section{Introduction}

Chronic kidney disease has been recognized as a major public health issue worldwide, ${ }^{1}$ and its prevalence in China has been increasing. ${ }^{2}$ Patients with stage 5 chronic kidney disease require renal replacement therapy for survival, and hemodialysis is the most commonly used therapy at present. ${ }^{3}$ Although hemodialysis can prolong the life of these patients, it also places a burden of many symptoms. Chronic pain is one of the most common symptoms of patients, the prevalence of chronic pain can be as high as $82 \%$ in MHD patients, ${ }^{4}$ and such pain severely affects quality of life (QOL). ${ }^{5}$

QOL is a multidimensional concept influenced by personal and environmental factors, as well as their interactions. Studies have reported poor QOL in maintenance hemodialysis (MHD) patients, especially those suffering from chronic pain. ${ }^{6,7}$ The QOL of these patients is associated with socio-demographic factors such as age, sex, level of education, and marital status, ${ }^{8-10}$ clinical characteristics such as primary disease, comorbidities, and duration of hemodialysis, ${ }^{11,12}$ as well as chronic pain factors including the duration of pain, pain severity, pain affect, the location of pain. ${ }^{5,13,14}$ Most importantly, psychosocial factors such as social support, self-efficacy, and depression, can also significantly affect QOL. ${ }^{15-17}$

Social support is defined as the interpersonal transaction involving one or more of the following: (1) emotional concern (liking, love, empathy), (2) instrumental aid (goods or services), (3) information (about the environment), or (4) appraisal (information relevant to self-evaluation). Several theories of behavioral change, including the Social Ecological Model, the Social Cognitive Theory and the Health Belief Model, consider social support to be a key factor that can improve QOL. ${ }^{18}$ Although several studies have shown that adequate social support can have a positive impact on the QOL of MHD patients with chronic pain, ${ }^{15,19}$ how social support affects QOL remains poorly understood.

Pain self-efficacy refers to a patient's confidence in his or her ability to tolerate pain and perform daily activities in spite of it. ${ }^{20,21} \mathrm{~A}$ growing body of research has demonstrated that pain self-efficacy can predict mental and physical health. ${ }^{22,23}$ Patients with chronic pain who have higher self-efficacy levels are more likely to perform health-related behaviors to alleviate the pain, which in turn leads to lower psychological stress and higher QOL. In contrast, those with low self-efficacy levels typically experience worse QOL and general health. Furthermore, according to the triadic model of reciprocal determinism in Social Cognitive Theory, ${ }^{24}$ there is a reciprocal relationship between environment (support, feedback), behavior (practice, self-efficacy), and individual (experience, cognition). Considering that pain self-efficacy is self-efficacy in the context of pain, we can reasonably assume that pain self-efficacy may be an important mediating factor between social support and QOL.

Few studies have explored the role of pain self-efficacy on social support and QOL in MHD patients with chronic pain. In fact, the prevalence and extent of chronic pain among MHD patients are unclear, as are the predictors of their QOL. Addressing these questions may help Medical staff develop effective non-drug interventions to improve their QOL. Therefore, in this study, we aimed to (1) understand the chronic pain status of Chinese MHD patients, (2) identify the predictors of QOL in MHD patients with chronic pain, and (3) examine relationships among pain self-efficacy, social support, and QOL.

\section{Methods \\ Study Design}

This multicenter, cross-sectional study was conducted between October 2020 and April 2021. We included MHD patients with chronic pain who had undergone treatment in hemodialysis units at nine hospitals in Chengdu, China. The participants originated from different districts across Chengdu, thus representing a wide geographic spread. This study was approved by the Ethics Committee of the First Affiliated Hospital of Chengdu Medical College (2020CYFYIRB-BA-108), and it was conducted based on the principles in the Declaration of Helsinki.

\section{Participants and Procedure}

Patients were included if they (1) were at least 18 years old and could communicate effectively (verbally or in writing), (2) had received hemodialysis for at least three months and were in a stable condition, and (3) met the diagnostic criteria of chronic pain outlined by the International Association for the Study of Pain (IASP). ${ }^{25}$ According to these criteria, pain had to occur daily or 
almost daily, it had to last longer than 3 months, and the patient had to rate it with a score $\geq 3$ on a visual analog scale.

We provided relevant information on the purpose and content of this study to all included patients. Questionnaires were distributed after obtaining written informed consent from the participants. If participants had questions about the study, we provided a detailed clarification in a timely manner during completion of the questionnaire. In case the patient could not read or write, the researchers read out the questions word for word and recorded the patient's answers. The questionnaires were distributed and retrieved by a master's degree candidate and three undergraduate students who were part of the research team.

\section{Instruments}

Based on expert consultation and a review of relevant literature, we designed a questionnaire to collect demographic and clinical information about the participants. Demographic characteristics included age, sex, marital status, place of residence, level of education, employment status, and financial strain, while clinical characteristics included duration and frequency of hemodialysis, body mass index, number of comorbidities, and duration of pain. These data were collected through patient selfreport and verified by chart review.

We used the Brief Pain Inventory $(\mathrm{BPI})^{26}$ to assess pain interference and severity in the study participants. Severity was assessed as an average score based on four pain categories: worst, least, average, and current pain. Each category was scored on a scale ranging from 0 to 10 . Pain interference was assessed based on the extent to which pain interfered with general activity, walking ability, mood, sleep, work, interactions with other people, and enjoyment of life. The interference in each of these seven functional domains was scored on a scale from 0 to 10 , and the mean score across all seven domains was taken as the pain interference score. The BPI was also used to collect data on pain location, pain treatments or medication received, and the degree of pain relief at 24 $\mathrm{h}$ after treatment or medication. The BPI has shown good reliability and validity for assessment of chronic pain. ${ }^{27}$

The Medical Outcomes Study 36-item Short Form Health Survey (SF-36) has been widely used worldwide to assess QOL in general and specific populations. ${ }^{28} \mathrm{SF}-36$ is divided into two subscales: the Mental Component Summary (MCS) and the Physical Component Summary
(PCS). ${ }^{29}$ These subscales encompass eight QOL domains: physical functioning, role physical, bodily pain, general health, vitality, social functioning, role emotional, and mental health. The score for each domain was linearly converted to a standard score ranging from 0 to 100 , with higher score indicating better perception of health. ${ }^{30}$ In the present study, we used the Mainland Chinese version of the SF-36. This survey has shown good reliability and validity, and it can be applied to MHD patients. ${ }^{31,32}$

The Pain Self-Efficacy Questionnaire was originally developed to measure an individual's confidence while performing a range of activities. ${ }^{20}$ This questionnaire contains a total of 10 entries that are scored on the basis of a 7-point Likert scale, where 0 indicates "not at all confident" and 6 indicates "completely confident". The total possible score of the scale is 60 , and higher scores indicate greater pain self-efficacy. We used the Chinese version of the Pain Self-Efficacy Questionnaire, which has shown good reliability and validity. ${ }^{22}$

The Social Support Rating Scale was specifically designed to measure social support in the Chinese population, and it has shown good reliability and validity. ${ }^{33}$ This scale consists of 10 items that are used to determine three dimensions of social support: subjective support (4 items), objective support ( 3 items), and support usage (3 items). The total score can range from 12 to 66 ; the subjective support subscore, from 8 to 32; the objective support subscore, from 1 to 22; and the support usage subscore, from 3 to 12 . Higher scores indicate stronger social support.

\section{Data Analysis}

All statistical analyses were performed using SPSS 23.0 (IBM, Armonk, NY, USA). Continuous variables that were normally distributed were expressed as means and standard deviations, while categorical variables were expressed as numbers or percentages. Skewed data were expressed as medians and interquartile ranges (IQRs). We used independent-sample t-tests or analysis of variance to examine whether demographic and clinical characteristics varied significantly with MCS score, PCS score, pain selfefficacy, or social support. Results associated with $\mathrm{p}<0.05$ were considered statistically significant.

Pearson correlation analysis was performed to identify relationships among these variables. We used stepwise multiple regression to identify predictors of QOL in MHD patients with chronic pain. The mediation model was examined using the PROCESS 3.3 procedure with centered variables. In the regression analyses, parameter 
estimation was performed based on 5000 bootstrapped samples. When a bias corrected and accelerated $95 \%$ confidence interval (BCa 95\% CI) does not contain zero, the mediating role is statistically significant.

\section{Results}

Of the 1204 MHD patients who had undergone treatment in hemodialysis units at the nine study sites during the enrollment period, we excluded 882 because they did not meet the eligibility criteria. Of the remaining 322 patients, 26 (8.07\%) declined to participate in the study. Therefore, we included a total of 296 MHD patients with chronic pain in the study.

\section{Demographic and Clinical Characteristics}

Scores on MCS, PCS, Pain self-efficacy, and social support of MHD patients with chronic pain of different characteristics in Table 1. A majority $(60.8 \%)$ of the patients included in this study were $\geq 60$ years old (age range 2793 years). A large proportion of the patients were married (83.1\%), had completed a junior high School education or below (74.3\%), were not currently employed (97\%), and reported financial strain $(84.5 \%)$. We found that age, employment status, financial strain, and number of comorbidities were significantly associated with MCS, PCS, and pain self-efficacy scores $(\mathrm{p}<0.05)$.

\section{Chronic Pain}

Of the 1204 MHD patients screened in this study, we found that only $322(26.7 \%)$ reported chronic pain. Based on the BPI, the pain severity score was 3.6 (IQR 3-4.6) and the pain interference score was 4 (IQR 3-5). Additionally, the duration of chronic pain was determined to be 5 years (IQR 1-7 years). Pain interference across the seven functional domains is depicted in Figure 1.

The chronic pain reported by patients was located in the lower back (63.5\%), lower limbs (55\%), head (33.5\%), shoulders (28.4\%), and feet (27.4\%). A large proportion of patients $(71.3 \%)$ reported experiencing pain at two or more sites. Many patients perceived the most painful area to be the lower back (39.5\%), followed by the lower limbs (25\%).

\section{Pain Management}

$36.5 \%$ of patients did not take any measures to relieve pain, while $38.9 \%$ of these patients experienced severe pain (worst pain $\geq 7$ ). Among the patients who received treatments or medications for pain relief, 56.9\% reported that the measures they took had less than half of the pain relief. The main pain relief measures taken by the patients included traditional Chinese medicines (45.3\%), medication $(27.4 \%)$, and physiotherapy (3.7\%).

\section{Predictors of QOL}

Stepwise multiple linear regression was used to identify predictors of QOL, as measured in terms of MCS and PCS scores (Table 2). The independent predictors of MCS score were age $(p=0.004)$, financial strain $(p=0.001)$, pain interference $(p<0.001)$, social support $(p=0.023)$, and pain self-efficacy $(\mathrm{p}<0.001)$. These variables accounted for $57.3 \%$ of the variance in MCS scores (adjusted $R^{2}=$ $0.573)$.

Independent predictors of PCS score were age $(\mathrm{p}=$ $0.016)$, sex $(p=0.043)$, financial strain $(p=0.016)$, frequency of hemodialysis $(\mathrm{p}=0.027)$, pain interference $(\mathrm{p}<$ $0.001)$, social support $(p=0.019)$, and pain self-efficacy ( $p$ $<0.001)$. These variables accounted for $75.4 \%$ of the variance in PCS scores (adjusted $R^{2}=0.754$ ).

\section{Correlation Analysis}

We performed Pearson's correlation analysis to explore relationships among social support, pain self-efficacy, MCS and PCS. There was a positive correlation among all four variables. Means, standard deviations and correlations coefficient for each variable are shown in Table 3.

\section{Mediation Model Test}

After controlling for age, employment status, financial strain, and number of comorbidities as covariates, we found that pain self-efficacy mediated the relationship between social support and QOL, as measured in terms of MCS and PCS scores. The paths (a, b, c, and c') and the indirect path $\mathrm{a} * \mathrm{~b}$ are labeled with the path coefficients in the mediation model (Figure 2). Tables 4, 5 showed the results of the mediation analysis.

In model 1, the connection between social support and MCS (c path) was examined, social support has a positive relation with MCS $(\mathrm{c}=1.098, \mathrm{p}<0.001)$. Then, the indirect effect of social support on MCS via pain self-efficacy was observed (path $a^{*} b, \mathrm{a}=0.597, \mathrm{~b}=1.292$ ). Meanwhile, when pain self-efficacy was entered to the model as a mediator, the direct effect of social support on MCS (path $c^{\prime}$ ) was still significant $\left(\mathrm{c}^{\prime}=0.326, \mathrm{p}<0.05\right)$. In addition, the upper and lower limits of bootstrap 95\% confidence intervals for the direct effect of social support on MCS and the mediating effect of pain self-efficacy did 
Table I Scores on MCS, PCS, Pain Self-Efficacy, and Social Support of MHD Patients with Chronic Pain of Different Characteristics $(n=296)$

\begin{tabular}{|c|c|c|c|c|c|}
\hline \multirow[t]{2}{*}{ Variables } & Total Sample & MCS & PCS & Pain Self-Efficacy & Social Support \\
\hline & n (\%) & Mean (SD) & Mean (SD) & Mean (SD) & Mean (SD) \\
\hline \multicolumn{6}{|l|}{ Age (years) } \\
\hline $18-44$ & $23(7.8 \%)$ & $60.1(14.6)$ & $49.5(9.7)$ & $28.6(5.9)$ & $27.8(4.8)$ \\
\hline $45-59$ & 93 (31.4\%) & $54.5(17.3)$ & $46.7(13.3)$ & $25.0(8.6)$ & $28.0(5.2)$ \\
\hline$\geq 60$ & $180(60.8 \%)$ & $53.0(16.8)$ & $36.2(12.8)$ & 19.4 (7.7) & $26.8(6.1)$ \\
\hline$p$-value & & 0.032 & $<0.001$ & $<0.001$ & 0.204 \\
\hline \multicolumn{6}{|l|}{ Sex } \\
\hline Male & $126(42.6 \%)$ & $54.9(15.6)$ & $42.0(12.9)$ & $21.6(7.9)$ & $27.5(5.5)$ \\
\hline Female & $170(57.4 \%)$ & $51.6(17.5)$ & $39.5(14.4)$ & $22.0(8.9)$ & $27.0(5.9)$ \\
\hline$p$-value & & 0.095 & 0.133 & 0.659 & 0.520 \\
\hline \multicolumn{6}{|l|}{ Marital status } \\
\hline Married & 246 (83.1\%) & $53.1(16.8)$ & $41.0(13.5)$ & $22.1(8.4)$ & $27.8(4.8)$ \\
\hline Unmarried/ divorced/ widowed & $50(16.9 \%)$ & $52.2(16.8)$ & $38.2(15.3)$ & $20.3(9.0)$ & $24.4(8.6)$ \\
\hline$p$-value & & 0.715 & 0.193 & 0.167 & 0.010 \\
\hline \multicolumn{6}{|l|}{ Living place } \\
\hline Country & 50 (16.9\%) & $50.2(14.7)$ & $38.9(14.0)$ & $19.8(6.7)$ & $26.5(4.5)$ \\
\hline Town & 37 (I2.5\%) & $58.2(18.4)$ & $42.5(15.5)$ & $24.0(9.9)$ & $27.1(5.7)$ \\
\hline City & 209 (70.6\%) & $52.7(16.8)$ & $40.6(13.5)$ & $21.9(8.6)$ & $27.4(6.0)$ \\
\hline$p$-value & & 0.081 & 0.489 & 0.067 & 0.546 \\
\hline \multicolumn{6}{|l|}{ Education levels } \\
\hline Junior school or less & $220(74.3 \%)$ & $52.0(16.7)$ & $39.9(13.9)$ & $21.1(8.2)$ & $26.6(5.4)$ \\
\hline High school/technical secondary & $56(18.9 \%)$ & $56.0(17.3)$ & $43.4(14.3)$ & $24.0(9.4)$ & $28.9(6.4)$ \\
\hline College or above & $20(6.8 \%)$ & $55.0(15.5)$ & $41.2(10.7)$ & $24.0(8.0)$ & $29.6(6.14)$ \\
\hline$p$-value & & 0.246 & 0.221 & 0.045 & 0.004 \\
\hline \multicolumn{6}{|l|}{ Employment status } \\
\hline Full-time/part-time & $9(3.0 \%)$ & $65.2(12.1)$ & $52.0(12.3)$ & $30.1(7.8)$ & $30.0(5.3)$ \\
\hline Unemployed & 287 (97.0\%) & $52.6(16.8)$ & $40.2(13.7)$ & $21.6(8.4)$ & $27.2(5.7)$ \\
\hline$p$-value & & 0.025 & 0.012 & 0.003 & 0.196 \\
\hline \multicolumn{6}{|l|}{ Financial strain } \\
\hline None & 46 (15.5\%) & $63.0(13.8)$ & $48.0(12.5)$ & $24.7(8.4)$ & $30.9(7.3)$ \\
\hline Mild or moderate & $84(28.4 \%)$ & $55.0(15.7)$ & $40.4(12.3)$ & $21.8(8.8)$ & $27.2(6.0)$ \\
\hline Serious & $166(56.1 \%)$ & $49.0(16.7)$ & $38.6(14.3)$ & $21.1(8.2)$ & $26.3(4.6)$ \\
\hline$p$-value & & $<0.001$ & $<0.001$ & 0.040 & $<0.001$ \\
\hline \multicolumn{6}{|l|}{ Hemodialysis duration (years) } \\
\hline$<1$ & $26(8.8 \%)$ & $56.0(15.8)$ & $42.0(14.6)$ & $21.6(8.3)$ & $26.9(6.2)$ \\
\hline $1-5$ & $172(58.1 \%)$ & $51.4(17.0)$ & $40.0(14.2)$ & $21.1(8.6)$ & $27.3(5.7)$ \\
\hline$>5$ & $98(33.1 \%)$ & $54.9(16.6)$ & $41.3(13.0)$ & $23.1(8.3)$ & $27.2(5.7)$ \\
\hline$p$-value & & 0.166 & 0.646 & 0.167 & 0.935 \\
\hline \multicolumn{6}{|l|}{ Hemodialysis frequency } \\
\hline 2 times weekly & 29 (9.8\%) & $55.2(16.2)$ & 41.7 (15.9) & $21.1(9.0)$ & $27.7(7.1)$ \\
\hline 3 times weekly & $267(90.2 \%)$ & $52.7(16.8)$ & $40.4(13.6)$ & $21.9(8.5)$ & $27.2(5.6)$ \\
\hline$p$-value & & 0.447 & 0.633 & 0.652 & 0.683 \\
\hline \multicolumn{6}{|l|}{ BMI } \\
\hline$<18.5$ & 21 (7.1\%) & $52.9(15.2)$ & $42.6(12.1)$ & $22.7(5.7)$ & $27.6(7.2)$ \\
\hline $18.5-24.0$ & 187 (63.2\%) & $53.1(16.8)$ & $41.0(13.6)$ & 22.1 (8.6) & $27.1(5.6)$ \\
\hline$>24.0$ & 88 (29.7\%) & $52.7(17.3)$ & $39.2(14.6)$ & $21.0(9.0)$ & $27.2(5.7)$ \\
\hline$p$-value & & 0.986 & 0.487 & 0.508 & 0.931 \\
\hline \multicolumn{6}{|l|}{ Comorbidities } \\
\hline$<3$ & 124 (4I.9\%) & $56.3(15.7)$ & $44.9(12.1)$ & $24.4(8.8)$ & $28.0(5.4)$ \\
\hline$\geq 3$ & 172 (58.1\%) & $50.5(15.7)$ & $37.5(14.2)$ & $20.0(7.8)$ & $26.7(5.9)$ \\
\hline$p$-value & & 0.003 & $<0.001$ & $<0.001$ & 0.040 \\
\hline
\end{tabular}

Note: $p<0.05$ was considered statistically significant.

Abbreviations: MCS, mental component summary; PCS, physical component summary; BMI, body mass Index; SD, standard deviation. 


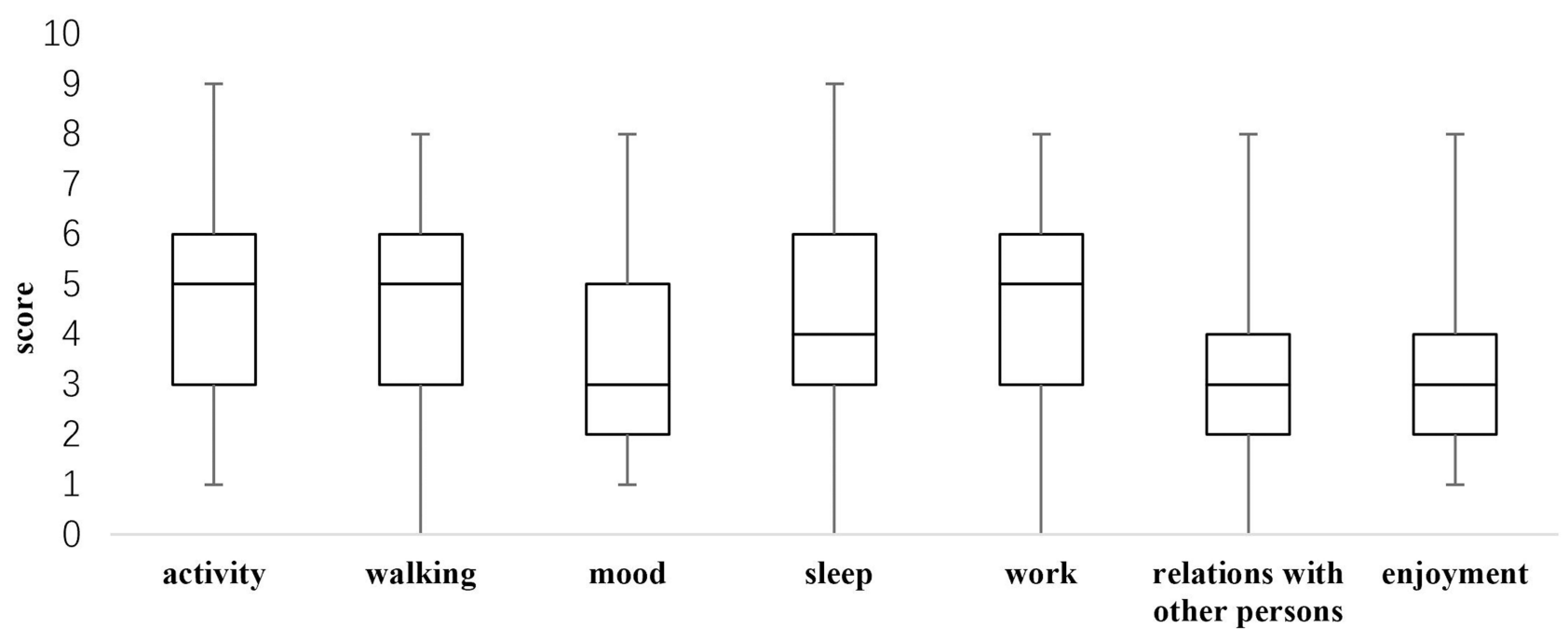

Seven functional domains of pain interference

Figure I Box plot of the seven functional domains scores of pain interference.

not contain zero. Therefore, pain self-efficacy had a partial mediating effect in the relationship between social support and pain self-efficacy for MHD patients with chronic pain. The direct effect $(0.33)$ and mediating effect $(0.77)$ accounted for $29.69 \%$ and $70.31 \%$ of the total effect, respectively.

Similarly, in model 2, social support has a positive relation with PCS $(\mathrm{c}=0.875, \mathrm{p}<0.001)$. The indirect effect of social support on MCS via pain self-efficacy (path $a^{*} b, \mathrm{a}=0.597, \mathrm{~b}=1.107$ ). Moreover, when pain self-efficacy was entered to the model as a mediator, the direct effect of social support on PCS (path c') was still significant $\left(c^{\prime}=0.213, p<0.05\right)$. The confidence interval for indirect effect did not contain zero, which suggested that pain self-efficacy played a mediating role between social support and PCS. The direct effect $(0.21)$ and

Table 2 Stepwise Multiple Regression to Identify Predictors of Quality of Life in MHD Patients with Chronic Pain

\begin{tabular}{|l|c|c|c|c|}
\hline \multirow{2}{*}{ Variables } & \multicolumn{2}{|c|}{ MCS } & \multicolumn{2}{c|}{ PCS } \\
\cline { 2 - 5 } & Coefficient $\boldsymbol{\beta}$ & p-value & Coefficient $\beta$ & p-value \\
\hline Age & 0.126 & 0.004 & -0.118 & 0.016 \\
Gender & -0.053 & 0.177 & -0.060 & 0.043 \\
Marital status & -0.032 & 0.419 & -0.012 & 0.698 \\
Living place & -0.044 & 0.260 & -0.010 & 0.736 \\
Education levels & -0.029 & 0.471 & -0.058 & 0.059 \\
Employment status & -0.027 & 0.502 & 0.249 \\
Financial burden & -0.142 & 0.001 & 0.035 & 0.016 \\
Hemodialysis duration & -0.013 & 0.739 & -0.077 & 0.775 \\
Hemodialysis frequency & -0.055 & 0.152 & -0.009 & 0.027 \\
BMI & 0.046 & 0.240 & -0.065 & 0.999 \\
Comorbidities & -0.019 & 0.638 & 0.000 & 0.166 \\
Chronic pain duration & -0.003 & 0.930 & -0.043 & 0.418 \\
Pain severity & -0.046 & 0.377 & 0.025 & 0.701 \\
Pain interference & -0.266 & $<0.001$ & 0.015 & $<0.001$ \\
Social support & 0.101 & 0.023 & -0.350 & 0.019 \\
Pain self-efficacy & 0.510 & $<0.001$ & 0.079 & $<0.001$ \\
\hline
\end{tabular}

Notes: Quality of life was measured based on the mental component summary score (MCS) and the physical component summary score (PCS); $p<0.05$ was considered statistically significant.

Abbreviations: MCS, mental component summary; PCS, physical component summary; BMI, body mass index. 
Table 3 Pearson Correlation Analysis of Relationships Among Social Support, Pain Self-Efficacy, MCS, and PCS in MHD Patients with Chronic Pain

\begin{tabular}{|l|c|c|c|c|c|}
\hline & Mean (SD) & I & $\mathbf{2}$ & $\mathbf{3}$ & $\mathbf{4}$ \\
\hline Social support & $27.23(5.74)$ & $\mathrm{I}$ & & & \\
Pain self-efficacy & $21.82(8.5 \mathrm{I})$ & $0.5^{* *}$ & $\mathrm{I}$ & & \\
MCS & $53(16.76)$ & $0.46^{* *}$ & $0.69 * *$ & $\mathrm{I}$ & \\
PCS & $40.56(13.8 \mathrm{I})$ & $0.48^{* *}$ & $0.8^{* *}$ & $0.78^{* *}$ & $\mathrm{I}$ \\
\hline
\end{tabular}

Notes: $p<0.05$ was considered statistically significant; ${ }^{* *} p<0.01$.

Abbreviations: MCS, mental component summary; PCS, physical component summary; SD, standard deviation.

mediating effect (0.66) accounted for $24.38 \%$ and $75.62 \%$ of the total effect.

\section{Discussion}

In the present study, the prevalence of chronic pain in MHD patients was $26.74 \%$. This value is lower than that reported in other studies, ${ }^{6,34,35}$ likely reflecting differences in pain assessment tools, inclusion criteria, as well as ethnic and cultural factors. ${ }^{4,36}$ Indeed, most previous studies have defined chronic pain primarily in terms of duration, whereas we applied three criteria based on frequency, duration and rating on a visual analogue scale.

The score for the pain severity score was 3.6 (IQR 3, 4.6) and pain interference was 4 (IQR 3, 5.0), chronic pain had different effects on the seven functional domains of pain interference, and the greatest interference on general activity and walking ability. Based on stepwise multiple regression, we found that pain interference had a negative association with QOL, and it independently predicted QOL in MHD patients. These results are consistent with another study ${ }^{37}$ reporting that pain interference can predict QOL of MHD patients better than pain severity. Most patients in the present study complained of pain in the lower back and lower limbs. Unlike chronic pain in the general population, chronic pain in MHD patients is more specific at the site and lasts longer. ${ }^{38}$ Further research should explore the associations among the characteristics of chronic pain, chronic renal failure, and hemodialysis.

In terms of pain management, we found that $36.5 \%$ of participants did not take any measures to relieve pain, while among the patients who received treatments or medications for pain relief, $56.9 \%$ thought that the analgesic effect of the measures they took were less than half, consistent with other studies. $^{34,39}$ Therefore, we conclude that pain is poorly managed in a large proportion of MHD patients with chronic pain in China. Traditional Chinese medicines may be useful in this context: they are known to effectively relieve chronic and neuropathic pain in patients with chronic kidney disease, ${ }^{40}$ and they were the pain treatment received by nearly half of those who received any pain treatment or medication in our study.

In this study, the score of MCS was $53 \pm 16.76$, PCS was $40.56 \pm 13.81$. Apparently, compared with the full score of 100, the QOL of MHD patients with chronic pain was at a low level. Moreover, we found that older age was

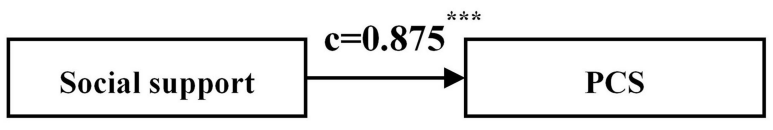

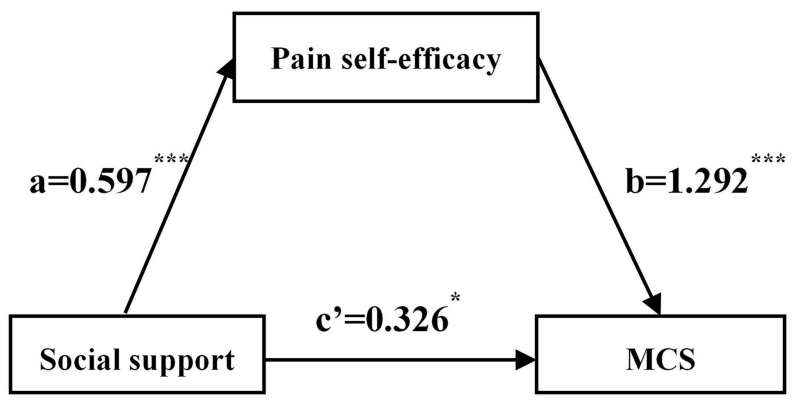

Model 1

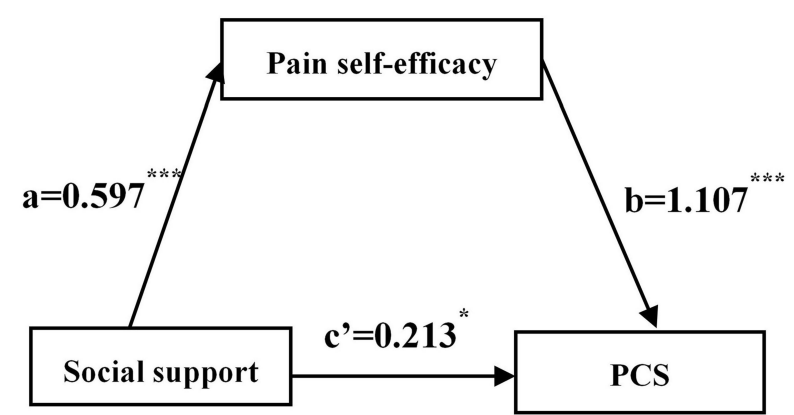

Model 2

Figure 2 Mediation model of the effects of pain self-efficacy on the relationship between social support and quality of life.

Notes: Quality of life was measured based on the mental component summary score (MCS) and the physical component summary score (PCS); ${ }^{*} p<0.05$, $* * * p<0.00 \mathrm{I}$. Abbreviations: MCS, mental component summary; PCS, physical component summary. 
Table 4 Mediation Model Test of Pain Self-Efficacy

\begin{tabular}{|c|c|c|c|c|c|c|c|}
\hline & \multicolumn{2}{|c|}{ Regression Equation } & \multicolumn{3}{|c|}{ Overall Model Fit } & \multicolumn{2}{|c|}{ Regression Coefficient } \\
\hline & Outcome & Predictor & $R$ & $R^{2}$ & $\boldsymbol{F}$ & $\beta$ & $t$ \\
\hline Model I & $\begin{array}{l}\text { Pain self-efficacy } \\
\text { MCS }\end{array}$ & $\begin{array}{l}\text { Age } \\
\text { Employment status } \\
\text { Financial strain } \\
\text { Comorbidities } \\
\text { Social support } \\
\text { Age } \\
\text { Employment status } \\
\text { Financial strain } \\
\text { Comorbidities } \\
\text { Pain self-efficacy } \\
\text { Social support }\end{array}$ & $\begin{array}{l}0.62 \\
0.73\end{array}$ & $\begin{array}{l}0.39 \\
0.54\end{array}$ & $36.93^{* * *}$ & $\begin{array}{l}-0.22 \\
-1.96 \\
-1.24 \\
-0.48 \\
0.60 \\
0.16 \\
-2.24 \\
-3.64 \\
-0.13 \\
1.29 \\
0.33\end{array}$ & $\begin{array}{c}-6.56^{* * *} \\
-0.83 \\
-2.17^{*} \\
-1.97^{*} \\
8.24^{* * *} \\
2.56^{*} \\
-0.55 \\
-3.70^{* * *} \\
-0.30 \\
12.86^{* * *} \\
2.37^{*}\end{array}$ \\
\hline Model 2 & $\begin{array}{l}\text { Pain self-efficacy } \\
\text { PCS }\end{array}$ & $\begin{array}{l}\text { Age } \\
\text { Employment status } \\
\text { Financial strain } \\
\text { Comorbidities } \\
\text { Social support } \\
\text { Age } \\
\text { Employment status } \\
\text { Financial strain } \\
\text { Comorbidities } \\
\text { Pain self-efficacy } \\
\text { Social support }\end{array}$ & 0.62 & 0.39 & $99.92 * * *$ & $\begin{array}{l}-0.22 \\
-1.96 \\
-1.24 \\
-0.48 \\
0.60 \\
0.16 \\
-2.24 \\
-3.64 \\
-0.13 \\
1.29 \\
0.33\end{array}$ & $\begin{array}{c}-6.56^{* * *} \\
-0.83 \\
-2.17^{*} \\
-1.97^{*} \\
8.24^{* * *} \\
-0.14^{* *} \\
2.05 \\
-2.41^{* * *} \\
-0.27 \\
1.11^{* * *} \\
0.21^{*}\end{array}$ \\
\hline
\end{tabular}

Notes: $p<0.05$ was considered statistically significant; $* p<0.05$, $* * p<0.0$ I, and $* * * p<0.00$ I; $\beta$, standardized regression coefficient; $R$, correlation coefficient; $R^{2}$, coefficient of determination; $F$, homogeneity of variance test; $t$, statistical value of $t$-test.

Abbreviations: MCS, mental component summary; PCS, physical component summary.

Table 5 Decomposition Table of Total, Direct and Mediating Effects

\begin{tabular}{|l|l|r|r|r|r|r|}
\hline & \multicolumn{1}{|c|}{ Effect } & Effect Size & Bootstrap Standard Error & LLCl & ULCI & Relative Effect Size \\
\hline Model I & Total & 1.1 & 0.16 & 0.8 & 1.4 & \\
& Direct & 0.33 & 0.14 & 0.05 & 0.6 & $29.69 \%$ \\
& Mediating & 0.77 & 0.15 & 0.5 & 1.09 & $70.31 \%$ \\
\hline \multirow{2}{*}{ Model 2 } & Total & 0.87 & 0.12 & 0.64 & 1.11 & \\
& Direct & 0.21 & 0.1 & 0.03 & 0.4 & $24.38 \%$ \\
& Mediating & 0.66 & 0.12 & 0.45 & 0.93 & $75.62 \%$ \\
\hline
\end{tabular}

Abbreviations: LLCI, lower limit of the confidence interval; ULCI, upper limit of the confidence interval.

associated with higher MCS score and lower PCS score. We speculate that older patients may have a greater sense of fulfillment, making them less worried about family, society and the future, which results in better overall mental health and therefore higher MCS scores. At the same time, older patients are more likely to experience physical ailments that can decrease their ability to take care of themselves, resulting in lower PCS scores.
In the present study, $74.3 \%$ of the patients had a junior high school education or below, $97.0 \%$ did not participate in work, and $84.5 \%$ reported economic pressure during hemodialysis. Meanwhile, the results of stepwise multiple regression models showed that financial burden was an independent predictor of QOL. This was consistent with the study of Modi et al. ${ }^{41}$ With the increase of medical insurance coverage in China, patients can reimburse most 
of expenses. However, for these patients with lower socioeconomic status, characterized by lower education, worse financial situation, or lack of employment, still cannot afford therapy, which can affect their adherence. ${ }^{42}$ Since patients have to undergo hemodialysis $2-3$ times a week for a duration of $4 \mathrm{~h}$ each time, they spend a lot of time and energy on therapy and may be forced to stop working due to the chronic pain. These factors can create significant mental and physical strain on MHD patients, thus affecting their QOL.

We also identified social support as an independent predictor of QOL in MHD patients with chronic pain. These results are consistent with previous studies reporting that patients with higher levels of social support experienced better mental and physical health. ${ }^{15,19}$ Patients who receive moral and material support from their families and society are more capable of psychological adjustment and are able to deal with negative events more constructively. ${ }^{43}$ In contrast, those with inadequate social support or those living in a stressful family environment are more likely to experience psychological problems such as low self-esteem and loss of self-identity. Such individuals are more likely to think negatively about their disease and treatment, which can ultimately affect QOL. We found higher levels of social support among married patients, suggesting that support from a partner can be a particularly important component of social support. Indeed, social support is associated with adherence to dietary restrictions, suggesting that family support can benefit physical health. ${ }^{44}$

We found that pain self-efficacy positively correlated with QOL among MHD patients with chronic pain. An important independent predictor of QOL, pain selfefficacy explained $47.4 \%$ of MCS and $64.4 \%$ of PCS in the present study. Individuals with high pain self-efficacy have stronger pain coping beliefs and are more likely to comply with health-related behaviors. ${ }^{45}$ One study also found that the level of pain self-efficacy is negatively associated with anxiety and depression, which may also affect the QOL. ${ }^{46}$

Finally, we detected positive correlations among social support, pain self-efficacy, MCS, and PCS. After controlling for clinicodemographic characteristics, we found that pain self-efficacy may partially mediate the relationship between social support and QOL. Meanwhile, the mediating effect value of pain self-efficacy in social support and MCS was $70.31 \%$, and the mediating effect value in social support and PCS was $75.62 \%$. The results suggested that while social support had an important role in improving patients' QOL, pain self-efficacy also played a critical mediating role in improving the patients' QOL. Possible explanation for this interaction is that social support may be an important element for patients to improve the QOL, but higher pain self-efficacy even gives them stronger beliefs to cope with pain, actively seek and utilize social support, and ultimately improve their QOL. There is growing evidence that pain self-efficacy is a predictor of physical and mental health, ${ }^{23,47}$ patients with high pain self-efficacy appear to be more active in using social support to promote their physical and mental health.

There are some limitations in the present study. First, our cross-sectional dataset did not allow us to evaluate longitudinal changes in social support, pain self-efficacy, or QOL. Second, we did not collect data to help identify the underlying causes of poor pain management in MHD patients. Qualitative studies are necessary in order to develop effective pain management strategies for such patients. Third, we did not collect data on certain psychosocial factors, such as depression or anxiety. Such factors should be considered when formulating pain management strategies. Finally, an exploration based on the etiology of chronic pain might contribute to more targeted chronic pain management strategies.

\section{Conclusion}

To conclude, chronic pain is common in MHD patients, especially in the elderly. Pain sites were most common in the lower back and lower limbs. Most patients in our study reported inadequate treatment and management of chronic pain. MHD patients with chronic pain had poor QOL, and QOL was significantly associated with age, financial strain, pain interference, social support, and pain self-efficacy. Based on the results of this study, we put forward the following suggestions. First, medical staff should pay more attention to the assessment and management of chronic pain among MHD patients. Appropriate pain management strategies are essential to meet the needs of this patient population. Second, the influence of psychosocial factors on MHD patients should be researched in detail. Social support and pain self-efficacy play important roles in improving the QOL of MHD patients with chronic pain. Improving social support, especially partner support, may help improve pain selfefficacy and therefore coping ability and treatment adherence. Psychological therapies such as health education, cognitive behavioral therapy, supportive psychotherapy 
and mindfulness therapy may be useful for improving pain self-efficacy and social support.

\section{Acknowledgments}

We would like to thank Xiaofang Chen $\mathrm{MPH} \mathrm{PhD}$ for assisting us with the statistical analyses.

\section{Funding}

This study was supported by the Key Projects of Chengdu Medical College Graduate Research Innovation Fund 2020 (YCX2020-12).

\section{Disclosure}

The authors declare no conflicts of interest in this work.

\section{References}

1. Lv JC, Zhang LX. Prevalence and disease burden of chronic kidney disease. Adv Exp Med Biol. 2019;1165:3-15.

2. Yang C, Yang Z, Wang J, et al. Estimation of prevalence of kidney disease treated with dialysis in China: a Study of Insurance Claims Data. Am J Kidney Dis. 2021;77(6):889. doi:10.1053/j.ajkd.2020.11.021

3. Zhang L, Wang J, Yang C, et al. International society of nephrology global kidney health atlas: structures, organization and services for the management of kidney failure in North and East Asia. Kidney Int Suppl. 2021;11(2):E77-E85. doi:10.1016/j.kisu.2021.01.011

4. Brkovic T, Burilovic E, Puljak L. Prevalence and severity of pain in adult end-stage renal disease patients on chronic intermittent hemodialysis: a systematic review. Patient Prefer Adherence. 2016;10:1131-1150. doi:10.2147/PPA.S103927

5. Samoudi AF, Marzouq MK, Samara AM, et al. The impact of pain on the quality of life of patients with end-stage renal disease undergoing hemodialysis: a multicenter cross-sectional study from Palestine. Health Qual Life Outcomes. 2021;19(1):39. doi:10.1186/s12955021-01686-z

6. Sadigova E, Ozkurt S, Yalcin AU. Pain assessment in hemodialysis patients. Cureus. 2020;12(2):e6903. doi:10.7759/cureus.6903

7. Rapti E, Damigos D, Apostolara P, et al. Patients with chronic pain: evaluating depression and their quality of life in a single center study in Greece. BMC Psychol. 2019;7(1):86. doi:10.1186/s40359-0190366-0

8. Mahato SKS, Apidechkul T, Sriwongpan P, et al. Factors associated with quality of life among chronic kidney disease patients in Nepal: a cross-sectional study. Health Qual Life Out. 2020;18:2071. doi:10.1186/s12955-020-01458-1

9. Lee J, Kim YC, Kwon S, et al. Impact of health-related quality of life on survival after dialysis initiation: a prospective cohort study in Korea. Kidney Res Clinical Practice. 2020;39(4):426-440. doi:10.23876/j.krcp.20.065

10. Marzouq MK, Samoudi AF, Samara A, et al. Exploring factors associated with pain in hemodialysis patients: a multicenter cross-sectional study from Palestine. BMC Nephrol. 2021;22:961. doi:10.1186/s12882-021-02305-1

11. Teles F, Amorim DAA, Freitas GLI, et al. Quality of life and depression in haemodialysis patients. Psychol Health Med. 2018;23 (9):1069-1078. doi:10.1080/13548506.2018.1469779

12. Khanh VDD, Hien TMN, Nhi THN, et al. Associations of socio-demographic, clinical and biochemical parameters with healthcare cost, health- and renal-related quality of life in hemodialysis patients: a Clinical Observational Study. Int J Env Res Pub He. 2020;17:655218.
13. Kesikburun B, Ekşioğlu E, Akdağ O, et al. Low back pain in hemodialysis patients: risk factors and its impact on health-related quality of life. Turk J Phys Med Rehabil. 2018;64(1):66-71. doi:10.5606/ tftrd.2018.1016

14. Fillingim RB, Loeser JD, Baron R, et al. Assessment of chronic pain: domains, methods, and mechanisms. J Pain. 2016;17(9 Suppl):T1020. doi:10.1016/j.jpain.2015.08.010

15. Alshraifeen A, Al-Rawashdeh S, Alnuaimi K, et al. Social support predicted quality of life in people receiving haemodialysis treatment: a cross-sectional survey. Nursing OPEN. 2020:1-9.

16. $\mathrm{Du} \mathrm{S}, \mathrm{Hu} \mathrm{L}$, Bai $\mathrm{Y}$, et al. The influence of self-efficacy, fear-avoidance belief, and coping styles on quality of life for Chinese patients with chronic nonspecific low back pain: a Multisite Cross-Sectional Study. Pain Pract. 2018;18(6):736-747. doi:10.1111/papr.12660

17. de Alencar SBV, de Lima FM, Dias LA, et al. Depression and quality of life in older adults on hemodialysis. Br J Psychiatry. 2020;42 (2):195-200. doi:10.1590/1516-4446-2018-0345

18. Lindsay SG, Banting L, Eime R, et al. The association between social support and physical activity in older adults: a systematic review. Int J Behav Nutr Phys Act. 2017;14(1):56. doi:10.1186/s12966-0170509-8

19. Pan KC, Hung SY, Chen CI, et al. Social support as a mediator between sleep disturbances, depressive symptoms, and health-related quality of life in patients undergoing hemodialysis. PLoS One. 2019;14(4):e0216045. doi:10.1371/journal.pone.0216045

20. Nicholas MK. The pain self-efficacy questionnaire: taking pain into account. Eur J Pain. 2007;11(2):153-163. doi:10.1016/j. ejpain.2005.12.008

21. Vergeld V, Utesch T. Pain-related Self-efficacy Among People With Back Pain: A Systematic Review of Assessment Tools. Clin J Pain. 2020;36(6):480-494. doi:10.1097/AJP.0000000000000818

22. Yang Y, Yang M, Bai J, et al. Validation of Simplified Chinese Version of the Pain Self-Efficacy Questionnaire (SC-PSEQ) and SC-PSEQ-2 for Patients With Nonspecific Low Back Pain in Mainland China. Spine. 2019;44(20):E1219-E1226. doi:10.1097/ BRS.0000000000003099

23. Duray M, Yagci N, Ok N. Determination of physical parameters associated with self-efficacy in patients with chronic mechanic low back pain. J Back Musculoskelet Rehabil. 2018;31(4):743-748. doi:10.3233/BMR-170993

24. Bandura A. Human agency in social cognitive theory. Am Psychol. 1989;44(9):1175-1184. doi:10.1037/0003-066X.44.9.1175

25. Aydede M. Does the IASP definition of pain need updating? Pain Rep. 2019;4(5):e777. doi:10.1097/PR9.0000000000000777

26. Cleeland CS, Ryan KM. Pain assessment: global use of the Brief Pain Inventory. Ann Acad Med Singap. 1994;23(2):129-138.

27. Poquet N, Lin C. The Brief Pain Inventory (BPI). J Physiother. 2016;62(1):52. doi:10.1016/j.jphys.2015.07.001

28. Yayikci YI, Karadag A. Effects of Conventional and Biological Drugs Used for the Treatment of Rheumatoid Arthritis on the Quality of Life and Depression. Eurasian J Med. 2019;51(1):12-16. doi:10.5152/eurasianjmed.2018.18018

29. Li J, Zhong D, Ye J, et al. Rehabilitation for balance impairment in patients after stroke: a protocol of a systematic review and network meta-analysis. BMJ Open. 2019;9(7):e026844. doi:10.1136/bmjopen-2018-026844

30. Ware JJ, Sherbourne CD, The MOS. 36-item short-form health survey (SF-36). I. Conceptual framework and item selection. Med Care. 1992;30(6):473-483. doi:10.1097/00005650-199206000-00002

31. Kusztal M, Nowak K, Magott-Procelewska M, et al. [Evaluation of health-related quality of life in dialysis patients. Personal experience using questionnaire SF-36]. Pol Merkur Lekarski. 2003;14 (80):113-117. Polish.

32. Li L, Wang HM, Shen Y. Chinese SF-36 Health Survey: translation, cultural adaptation, validation, and normalisation. $J$ Epidemiol Community Health. 2003;57(4):259-263. doi:10.1136/jech.57.4.259 
33. Cheng Y, Liu C, Mao C, et al. Social support plays a role in depression in Parkinson's disease: a cross-section study in a Chinese cohort. Parkinsonism Relat Disord. 2008;14(1):43-45. doi:10.1016/j. parkreldis.2007.05.011

34. Kliuk-Ben BO, Brill S, Sharon H. Chronic pain is underestimated and undertreated in dialysis patients: a retrospective case study. Hemodial Int. 2019;23(4):E104-E105. doi:10.1111/hdi.12736

35. Davison SN. The prevalence and management of chronic pain in end-stage renal disease. J Palliat Med. 2007;10(6):1277-1287. doi:10.1089/jpm.2007.0142

36. Upadhyay C, Cameron K, Murphy L, et al. Measuring pain in patients undergoing hemodialysis: a review of pain assessment tools. Clin Kidney J. 2014;7(4):367-372. doi:10.1093/ckj/sfu067

37. Rodriguez C, Ji M, Wang HL, et al. Cancer Pain and Quality of Life. $J$ Hosp Palliat Nurs. 2019;21(2):116-123. doi:10.1097/NJH.000 0000000000507

38. El-Metwally A, Shaikh Q, Aldiab A, et al. The prevalence of chronic pain and its associated factors among Saudi Al-Kharj population; a cross sectional study. BMC Musculoskelet Disord. 2019;20 (1):177. doi:10.1186/s12891-019-2555-7

39. Fleishman TT, Dreiher J, Shvartzman P. Pain in Maintenance Hemodialysis Patients: a Multicenter Study. J Pain Symptom Manage. 2018;56(2):178-184. doi:10.1016/j.jpainsymman.2018.05.008

40. Li SH, Li L, Yang RN, et al. Compounds of traditional Chinese medicine and neuropathic pain. Chin J Nat Med. 2020;18(1):28-35. doi:10.1016/S1875-5364(20)30002-9
41. Modi GK, Yadav AK, Ghosh A, et al. Nonmedical Factors and Health-Related Quality of Life in CKD in India. Clin J Am Soc Nephrol. 2020;15(2):191-199. doi:10.2215/CJN.06510619

42. Kefale B, Alebachew M, Tadesse Y, et al. Quality of life and its predictors among patients with chronic kidney disease: a hospital-based cross sectional study. PLoS One. 2019;14(2): e02121842. doi:10.1371/journal.pone.0212184

43. Zhang H, Zhao Q, Cao P, et al. Resilience and Quality of Life: exploring the Mediator Role of Social Support in Patients with Breast Cancer. Med Sci Monit. 2017;23:5969-5979. doi:10.12659/ MSM.907730

44. Sousa H, Ribeiro O, Paúl C, et al. Social support and treatment adherence in patients with end-stage renal disease: a systematic review. Semin Dial. 2019;32(6):562-574. doi:10.1111/sdi.12831

45. Hashimoto A, Sonohata M, Mawatari M. The Use of Oral Analgesics and Pain Self-Efficacy Are Independent Predictors of the Quality of Life of Individuals with Rheumatoid Arthritis. Pain Res Manag. 2020;2020:7409396. doi:10.1155/2020/7409396

46. Skidmore JR, Koenig AL, Dyson SJ, et al. Pain self-efficacy mediates the relationship between depressive symptoms and pain severity. Clin J Pain. 2015;31(2):137-144. doi:10.1097/AJP.0000000000000094

47. Wittkopf PG, Johnson MI. Mirror therapy: a potential intervention for pain management. Rev Assoc Med Bras. 2017;63(11):1000-1005. doi:10.1590/1806-9282.63.11.1000

\section{Publish your work in this journal}

The Journal of Pain Research is an international, peer reviewed, open access, online journal that welcomes laboratory and clinical findings in the fields of pain research and the prevention and management of pain. Original research, reviews, symposium reports, hypothesis formation and commentaries are all considered for publication. The manuscript management system is completely online and includes a very quick and fair peer-review system, which is all easy to use. Visit http:// www.dovepress.com/testimonials.php to read real quotes from published authors. 